\title{
Phoneme perception in a neonate with a left sylvian infarct
}

\author{
G. Dehaene-Lambertz, ${ }^{\mathrm{a}, \mathrm{b}, *}$ M. Pena, ${ }^{\mathrm{a}}$ A. Christophe, ${ }^{\mathrm{a}}$ and P. Landrieu ${ }^{\mathrm{b}}$ \\ ${ }^{a}$ Laboratoire de Sciences Cognitives et Psycholinguistique (EHESS, ENS \& CNRS UMR 8554), Paris, France \\ ${ }^{\mathrm{b}}$ Service de Neuropédiatrie, Centre Hospitalier Universitaire Bicêtre (AP-HP), France
}

Accepted 1 July 2003

\begin{abstract}
We report the case of a neonate tested three weeks after a neonatal left sylvian infarct. We studied her perception of speech and non-speech stimuli with high-density event-related potentials. The results show that she was able to discriminate not only a change of timbre in tones but also a vowel change, and even a place of articulation contrast in stop consonants. Moreover, a discrimination response to stop consonants was observed even when syllables were produced by different speakers. Her intact right hemisphere was thus able to extract relevant phonetic information in spite of irrelevant acoustic variation. These results suggest that both hemispheres contribute to phoneme perception during the first months of life and confirm our previous findings concerning bilateral responses in normal infants.
\end{abstract}

(C) 2003 Elsevier Inc. All rights reserved.

Keywords: Brain specialization; Left lesion; Phoneme; Infant; ERP; Language; MMN

\section{Introduction}

In most adults, language is mainly processed in the left hemisphere. Neuropsychology has acknowledged this fact for well over a century and modern brain imaging techniques have more recently confirmed it (Dehaene et al., 1997; Kim, Relkin, Lee, \& Hirsch, 1997). How this left specialization for language develops is not yet understood. A first line of hypotheses postulates that both hemispheres are initially equipotential and that the asymmetry observed in adults arises during development. In this context, several models have been proposed that disagree about the age at which the asymmetry emerges or is fully stabilized [anywhere from a few years of age to puberty (Bates, Vicari, \& Trauner, 1999; Lenneberg, 1967)]. They also diverge by the proposed mechanisms leading to hemispheric asymmetries. Some authors emphasize the structural changes that occur in the brain during development. They maintain that differences in maturation between the left and the right hemispheres at critical times may affect the localization of some cognitive functions such as face or speech

\footnotetext{
${ }^{*}$ Corresponding author. Fax: +33-1-45-21-32-31.

E-mail address: ghis@1scp.ehess.fr (G. Dehaene-Lambertz).
}

processing (Best, 1988; Corballis, 1991). Other authors insist that the physical characteristics of speech stimuli determine hemispheric asymmetry. Because of differences in the left and right auditory pathways, e.g. fibers size, myelinization, etc.., the fast temporal events contained in speech are better processed by the left hemisphere (Zatorre, Belin, \& Penhune, 2002). In most of these models, left-hemisphere specialization for language is a by-product of interactions between general mechanisms of brain development and the human environment.

A second and very different set of hypotheses speculates that language is a genetically encoded characteristic of homo sapiens-sapiens and that innate neuronal networks dedicated to language (processing and learning) are implemented in the brain from the outset (Mehler \& Dupoux, 1994; Pinker, 1994). These networks are supposedly found in infants and adults alike. Since adults typically show left-hemisphere specialization for speech processing, and since infants have very early speech processing capacities, infants are expected to demonstrate a left-hemisphere advantage as well (Bertoncini et al., 1989; Molfese \& Molfese, 1979).

In order to decide between the two preceding sets of hypotheses, it is necessary to study the organization of 
the brain before intensive exposure to speech has taken place. Between the numerous processes involved in language comprehension and production, phoneme perception is particularly suited for this purpose. On the one hand, identical functional abilities for phoneme perception have been found for adults and infants. Both infants and adults perceive phonemes categorically along acoustic dimensions such as the VOT (Eimas, Siqueland, Jusczyk, \& Vigorito, 1971), are able to normalize across irrelevant acoustic variations such as different voices or pitches (Kuhl, 1983), display duplex perception (Eimas \& Miller, 1992), and so on. On the other hand, phoneme perception relies on a well localized left-lateralized temporo-parietal network in adults. More frequent and more severe impairments in phoneme perception are observed after left rather than after right hemispheric lesions (Blumstein, Baker, \& Goodglass, 1977; Chobor \& Brown, 1987; Sidtis \& Volpe, 1988). When two different sounds are presented simultaneously, one to each ear (dichotic listening), normal adults usually show an advantage for processing words and phonemes presented to the right ear (thus preferentially processed by the left hemisphere) while timbre discrimination is facilitated for sounds presented to the left ear. Finally, imaging studies using MEG (Näätänen et al., 1997) or fMRI (Binder et al., 2000; Celsis et al., 1999; Jacquemot, Pallier, LeBihan, Dehaene, \& Dupoux, submitted) have confirmed functional asymmetry in phonological processing between the left and right cortices in normal subjects. Thus, phoneme perception can be used to study whether the same behavior is sustained by the same cerebral organization in infants and adults.

In parallel with the adult work, researchers have been studying young patients and normal infants using dichotic listening and neuro-imaging techniques. Neuropsychological data obtained with children are less informative than those from adults because phoneme perception has not been directly evaluated after a brain lesion in infants. Because of the difficulties associated with testing young children, language is usually evaluated years after the lesion onset; furthermore, evaluation during the first years of life consists either of recording free speech samples or of questionnaires filled out by parents concerning their children's language comprehension and production. These evaluations show that the consequences of early brain damage on language abilities are very different from those described for adults. First, lesions on both sides can impoverish language capacities. However, Aram et al. demonstrate that deficits differ depending on the side of the lesions (Aram \& Ekelman, 1986; Aram, Meyers, \& Ekelman, 1990). Second, young patients with a left lesion generally acquire fairly adequate language, even if their linguistic skills are at the lower end of the scale when compared to intact children (see Bates \& Roe, 2001 for a complete review of these studies). Thus, deficits are generally less severe than what is reported for adults, but children who have suffered an acute lesion are evaluated later than adults and recovery processes may already have occurred. It could be that language-specialized networks are initially located in the left hemisphere but that greater plasticity in immature brains allows for more efficient right-hemisphere substitution than in adults when the left hemisphere is damaged.

Using dichotic listening, Glanville, Best, and Levenson (1977), and Bertoncini et al. (1989) reported that very young infants display a right-ear advantage for dichotically presented syllables and a left-ear advantage for musical tones. However, two other studies failed to find a right-ear advantage for speech at a young age (Best, Hoffman, \& Glanville, 1982; Vargha-Khadem \& Corballis, 1979). These contradictory results may be due in part to the lack of sensitivity that characterizes dichotic listening (in right-handed adults, the magnitude of the right-ear advantage for speech varies from 60 to $80 \%$ across studies, Ahoniska, Cantell, Tolvanen, \& Lyytinen, 1993) and in part to the problems associated with running behavioral experiments on infants.

Using event-related potentials (ERPs), Molfese and Burger-Judisch (1991) observed larger ERPs on a right temporal electrode compared to the symmetrical left electrode for a phoneme change along the voice onset time dimension, but a reverse asymmetry for a change along the place of articulation dimension. Novak et al. (Novak, Kurtzberg, Kreuzer, \& Vaughan, 1989) recorded ERPs of greater amplitude on a central right electrode compared to the left when infants listened to syllables. However, because of the small number of electrodes used in both studies, it is difficult to conclude anything about lateralization. Indeed, a source located in the left hemisphere and directed transversely may generate a potential on the right side of the head and vice versa. More recently, using a high-density recording system (65 electrodes), Dehaene-Lambertz (2000) compared electrical responses to speech and non-speech stimuli in awake four-month-old infants and observed higher voltages over the left hemisphere than over the right for both syllables and continuous tones. The mismatch response to a change of phoneme peaked over the frontal and temporo-parietal electrodes. Its topography on the scalp was different from the mismatch response to a change of timbre that peaked over the fronto-temporal and central electrodes. However, there was no difference in left-right asymmetry for the linguistic and the nonlinguistic contrasts. There was therefore no evidence in favor of a greater left hemisphere involvement in linguistic processing compared to other types of auditory processing. These electrophysiological results are not fully conclusive, however, for several reasons: First, stimuli were presented binaurally in these infant studies, while in adults a right-ear advantage is observed only 
when stimuli are presented dichotically, a procedure in which stimuli enter into competition (O'Leary et al., 1996). Second, many brain-imaging experiments in adults have shown that it is easier to demonstrate a left ear advantage in phoneme perception, when subjects are paying attention to the task (Binder et al., 2000; Imaizumi et al., 1997). This was not the case in the infant ERP experiments, whereas it was the case in the behavioral experiments. Third, even with a large number of electrodes, it remains difficult to locate the generators of the electrical responses and this may complicate the interpretation of voltage asymmetry on the scalp.

Although their aim was not to study phoneme perception, two recent studies used imaging techniques with higher spatial resolution than ERPs. One relied on functional magnetic resonance imaging with threemonth-old infants (Dehaene-Lambertz, Dehaene, \& Hertz-Pannier, 2002) while the other used near infra-red spectroscopy on neonates (Pena et al., in press). In both experiments, 20-s periods of normal and reversed speech alternated with silent periods. Greater activity was observed over the left hemisphere than over the right. Although activity was more intense for normal speech than for reversed speech in the left temporal lobe, there was no significant interaction between the type of speech and the hemisphere.

To summarize, brain-imaging studies using infants suggest that both hemispheres are not equivalent, but rather that asymmetries are present very early on. The left hyper-reactivity observed both in ERPs and fMRI studies is not a general asymmetry between hemispheres but is related to auditory tasks. Measured at rest, the cerebral blood flow is symmetric during the first year of life. It then shows a right-hemisphere predominance between the ages of 1 and 3 and a shift in favor of the left after the age of 3 (Chiron et al., 1997). Awake two-month-old infants listening to their native language, exhibit significant asymmetry in the right frontal lobe (Dehaene-Lambertz et al., 2002). During visual tasks (diode and face perception), activation is predominant in the right infero-temporal gyrus in two-month-old infants (Tzourio-Mazoyer et al., 2002). Nc, an ERP component sensitive to familiarity, is asymmetric for faces at 6-months of age (de Haan \& Nelson, 1999). Thus left/right asymmetries depend on the tasks and the cortices involved. We do not yet know if these asymmetries are only biases to the left for auditory cortices and to the right for visual cortices; they may be increased by exposure to speech and faces and lead to the significant processing asymmetries observed in adults. Alternatively, it could be that the opposite hemisphere is unable to support the appropriate function in infants, as is the case in adults. Early neuropsychological observations that assess the capacities of the other hemisphere should help to answer this question.
Here, we report the case of an infant (L.G.) with a widespread left lesion at birth who was tested between 3 and 6 weeks afterward the lesion. Using high-density ERP, we were able to record her electrophysiological responses to speech and non-speech stimuli. By recording over several sessions, we obtained enough data to compute the statistical analyses usually performed in group studies of normally developing infants and were able to compare her discrimination capacities to those of normal neonates. Our goal was to determine whether a left neonatal lesion impairs phonetic discrimination as compared to other aspects of auditory processing, when auditory processing is measured right after the lesion has occurred. If this is the case, we can conclude that the left temporal lobe is necessary for phoneme perception in neonates, as it is in most adults. In a first experiment, we compared this infant's perception of auditory stimuli generally associated with the left hemisphere in adults, i.e., a place of articulation contrast /ba/ /ga/, and her perception of stimuli associated with the right hemisphere, i.e., a timbre contrast in two continuous tones. In a second experiment, we sought to ensure that L.G.'s capacity to discriminate consonants was a genuine linguistic capacity. The syllables used in Experiment 2 were thus produced by different speakers. Because of the irrelevant acoustical variation introduced by the change of speakers, a discrimination response to a consonant change could only be recorded if an abstract normalized representation of the phonemes was computed.

\section{Case report}

L.G. is the third daughter of French, right-handed, healthy parents. After an unproblematic pregnancy correctly supervised by local obstetricians, labor began on November 7th, 1998 at term (39 weeks 5 days of gestation). The labor was difficult: The fetus was in a shifted position and was larger than normal (all parameters at birth were superior to the $95^{\mathrm{e}}$ percentile: $4530 \mathrm{~g}, 54 \mathrm{~cm}$, cranial perimeter $=37 \mathrm{~cm}$ ). An emergency caesarian was performed due to increasing fetal distress, manifested by fetal cardiac rhythm disturbance and colored amniotic liquid. Although the APGAR score was 8 at 1', 9 at 5', and 10 at 10', signs of central nervous depression were immediately noted with global hypotonia and hyporeactivity. Three days after birth, abnormal movements of the right arm were observed with bursts of spikes recorded over the left hemisphere on an EEG. A large hypodensity area was visible over the left hemisphere on a CT scan. Therapy using phenobarbital was started immediately. At 17 days, L.G. who had been transferred to a neuropediatric unit, was lethargic, with no eye contact, little spontaneous motor activity and pronounced axial hypotonia. No neonatal reflexes (Moro, stepping, placing, tonic neck, palmar 

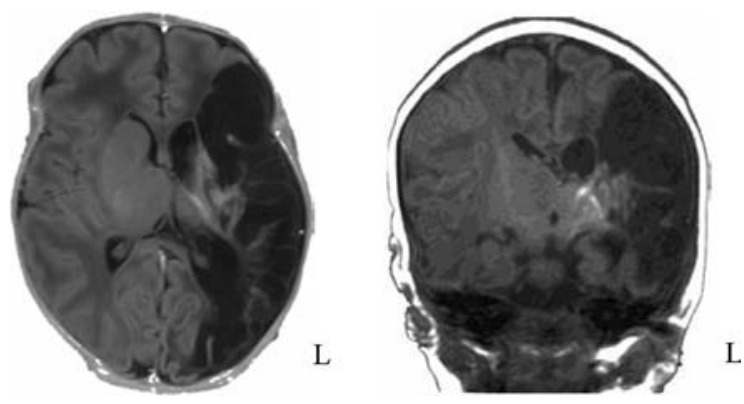

Fig. 1. Axial and coronal slices of a $\mathrm{T} 1$ sequence of magnetic resonance imaging performed at 3 weeks of age showing L.G.'s left sylvian infarct.

grasp) were present except for a weak sucking reflex. The electrophysiological activity was weak and discontinuous over the left hemisphere with sporadic spikes. No epileptic crisis was recorded. An MRI confirmed the recent left sylvian infarct (Fig. 1) involving the cortex, the basal ganglia and the white matter of the entire territory of the left middle cerebral artery. The left lateral ventricle was enlarged. Angiography sequences revealed an important decrease of flow in this artery. The flow in the other cerebral arteries was symmetric. At 6 months, an MRI with angiography sequences confirmed the remains of a large left ischemic lesion and the absence of the left middle cerebral artery. To summarize, L.G. presented a neonatal left sylvian infarct in a fullterm neonate occurring during delivery, with no evidence of more diffuse anoxic encephalopathy.

\section{Experiment 1: Responses to different types of auditory stimuli}

In the first experiment, we compared L.G.'s perception of auditory stimuli classically associated to the left and to the right hemispheres, i.e., phonemic discrimination vs timbre discrimination. In adults, a left temporal lesion is typically associated with difficulties in identifying and discriminating phonemes. In contrast, spectral information processing, controlled by the right hemisphere, is typically not impaired (Chobor \& Brown, 1987). Therefore, in the first half of Experiment 1, we contrasted discrimination of the timbre of two tones (i.e., differing in number of harmonics) and discrimination of a place of articulation contrast $/ \mathrm{ba} / / \mathrm{ga} /$. We chose this particular consonantal contrast because place of articulation is one of the phonemic contrasts that induces the greatest difficulties in language-impaired children (Kraus et al., 1996) or brain-lesioned adults (Miceli, Caltagirone, Gainotti, \& Payer-Rigo, 1978). Furthermore, processing this contrast requires an analysis of a fast-changing acoustic signal, a property that is usually attributed to the left hemisphere in adults. Thus, if infants' lateralization patterns are similar to those of adults', a discrimination response should be observed for the timbre change that requires only a spectral analysis, but not for the consonantal change.

To further refine the contrast between spectral and fast-temporal information processing, we compared consonants and vowels in the second half of Experiment 1. Although consonants and vowels are phonemes, the acoustical features crucial for their identification are different. Vowels last a few hundred milliseconds and their perception relies on spectral analyses of their constituting formants. Consonants, especially occlusives such as $/ \mathrm{b} /$ and $/ \mathrm{g} /$, are brief and their perception requires an analysis of fast-changing transitions. Dissociations in the impairment of vowel and consonant perception have been described in adults, following either a brain lesion or electrical stimulation (Boatman, Hall, Goldstein, Lesser, \& Gordon, 1997) and it is classically assumed that vowel processing is less leftlateralized than consonant processing. In the second half of Experiment 1, we thus compared discrimination responses to the same consonantal contrast (/ba/ vs /ga/) with a vowel contrast $(/ \mathrm{a} / \mathrm{vs} / \mathrm{o} /)$, in order to investigate the possibility that vowel discrimination would be less affected by a left lesion than consonant discrimination.

\subsection{Method}

Parents were informed of the study and provided their written informed consent. The study was approved by the local ethics committee (CCPPRB Paris Cochin).

\subsubsection{Stimuli}

Two sinewave tones were synthesized at a sampling rate of $16 \mathrm{kHz}$ using sound editing software (CoolEdit) with the same base frequency of $330 \mathrm{~Hz}$, the same duration of $285 \mathrm{~ms}$ and the same intensity of $78 \mathrm{~dB}$ SPL. The two tones differed in their number of harmonics: 5 for tone 1 and 2 for tone 2. Such a change in spectrum is perceived as a change of timbre.

Two syllables (/ba/ and /ga/), naturally produced by a French woman, were digitized at $16 \mathrm{kHz}$ and matched for total duration $(285 \mathrm{~ms})$, prevoicing and voiced formant transition duration ( 40 and $45 \mathrm{~ms}$, respectively). Two vowels $(/ \mathrm{a} /$ and $/ \mathrm{o} /)$ were produced by the same speaker. Duration and intensity of the linguistic stimuli were matched with the tones.

\subsubsection{Procedure}

2.1.2.1. Experimental design. We used a variant of the oddball design, that we have successfully used since 1994 to study infant auditory perception (Dehaene-Lambertz, 2000; Dehaene-Lambertz \& Baillet, 1998; DehaeneLambertz \& Dehaene, 1994). In our design, the stimuli are presented in trials of four stimuli each, with a within-trial stimulus onset asynchrony of $600 \mathrm{~ms}$ and an 
inter-trial interval of $4 \mathrm{~s}$. Two types of trials, standard and deviant, are randomly presented. In standard trials, the four stimuli are identical (A A A A). In deviant trials, the last stimulus of the trial is different from the first three (A A A B). Here, the standard stimulus was kept constant within a block of 70 trials to increase the probability of recording a mismatch response (Winkler, Cowan, Csépe, Czigler, \& Näätänen, 1996). In the next block, the standard stimulus became deviant and the deviant became standard, each stimulus successively serving as standard and deviant during a recording session. Stimulus randomization, presentation and synchronization with the ERP recording system were carried out using the EXPE software (Pallier, Dupoux, \& Jeannin, 1997) on a PC compatible with a Proaudio Spectrum 16 D/A Board.

Our design has two advantages over the classical oddball design. First, we can evaluate how the brain reacts to repetition and detects that two stimulus are similar by analyzing the response to the first three stimuli of the trials. Second, the introduction of the deviant stimulus is better controlled (it is always preceded by three standard stimuli) and the response to the same syllable at the same position in the trial is compared in standard and deviant trials. This avoids two confounds of the classical oddball design: If standard (i.e., /ba/) and deviant stimuli (i.e., /da/) presented in the same run are compared, the confound is that different waveforms may be observed due to acoustical differences between stimuli rather than to differences in phoneme representation. If responses to the same stimulus are compared when it is presented alone and when it is presented as a deviant in an oddball design, the confound is time and a potential habituation of low-level processing, or any change in the listening strategy that subjects might adopt even during a passive task.

\subsubsection{ERP recording and processing. ERPs were col-} lected using a 64-channel geodesic electrode net referred to the vertex. The net was placed on the sleeping baby who was seated in her mother's lap, facing two speakers. The mother cupped the infant's chin in her hand and kept the infant leaning slightly forward, so that the electrodes would not be in contact with anything. Due to her recent lesion, L.G. was in a stupor and could not be awoken for the ERP recording sessions. In adults, mismatch responses, i.e., the response to a change of stimulus in a series of repeated stimuli, have been recorded during all stages of sleep (Nielsen-Bohlman, Knight, Woods, \& Woodward, 1991) and even in some comatose patients (Fischer et al., 1999). Dehaene-Lambertz and Pena (2001) and Cheour et al. (2002a) have recorded mismatch responses to change of phonemes in sleeping neonates.

EEG activity was digitized continuously at $125 \mathrm{~Hz}$ then segmented over a $3152 \mathrm{~ms}$ epoch (a trial duration) including a $152 \mathrm{~ms}$ baseline. Channels contaminated by eye or motion artifacts were automatically rejected and trials with more than 25 contaminated channels were rejected. The remaining trials were averaged for each block and each of the different conditions, baselinecorrected and digitally filtered between 0.5 and $20 \mathrm{~Hz}$. An average reference transformation was applied to obtain the absolute potential. Two-dimensional reconstructions of scalp voltage for each sample were then computed using spherical spline interpolation on the grand-average across the different conditions.

L.G.'s ERPs were recorded in 6 sessions of about $30 \mathrm{mn}$ each (from 20 days of age to 1-month). Each session was made up of four blocks of 70 trials alternating between blocks of syllables testing the /ba/ /ga/ consonant discrimination and blocks of tones testing timbre discrimination for the first 3 sessions. In the last three sessions, the blocks with tones were replaced by blocks testing vowel discrimination $/ \mathrm{a} / \mathrm{o} /$. Thus, 12 blocks were obtained to study consonant perception, 6 blocks for vowel perception and 6 for tone perception. After artifacts rejection, the remaining trials were averaged for the standard and deviant conditions in each block. An average of 31.2 trials was kept in each condition and each block.

2.1.2.3. ERP analyses. Event-related responses can be characterized as electric dipoles, revealed by positivities and negativities on the scalp. To determine the peak latency of these responses, we computed the global field power of the voltage at each time point. To this end, we calculated the standard deviation across all channels in the grand-average at each time-sample. When no eventrelated response is present, all channels are around 0 , yielding a small standard deviation. In contrast, when an event-related response is present, the differences in voltage between channels increase, leading to an increase in global field power. We then determined the latency of the maximum global field power. This way of studying response latencies is conservative because it does not rely on any a priori hypothesis about the topography of the responses. This is especially useful in the present case since the presence of a brain lesion can affect the sources or the diffusion of brain activity through the tissues.

Two different effects were statistically analyzed. First, we analyzed the habituation effect, or how the repetition of the same stimulus was perceived. Clusters of 5 or 6 electrodes located at the dipole maxima on the two-dimensional reconstructions of scalp voltage of the grand average were selected. The voltage recorded at these locations was averaged for each block across a $80 \mathrm{~ms}$ time window centered on the peak maximum latency (determined as indicated above) and entered in an analysis of variance (ANOVA) with the following within-block factors: Electrodes (clusters at the positive maximum and at the negative maximum), Hemisphere (left and right), and Number of stimulus (1,2, and 3), as 
well as a between-blocks factor: Type of stimuli (e.g. Tones vs Vowels vs Stop consonants in Experiment 1). We expected a significant interaction between Electrode and Number of stimuli if habituation occurred. We also expected the Electrode by Hemisphere interaction to be significant because of the low voltage above the pathological left hemisphere.

Second, we analyzed the mismatch effect, or how the change in the 4th stimulus was perceived. An ANOVA was thus computed with the within-blocks factors Electrodes, Hemisphere, Condition (standard vs deviant) and the between block factor Type of stimuli. If a mismatch effect was present, the interaction between Electrodes and Condition should be significant. Since in our experimental design, stimuli were alternatively standard and deviant, a significant interaction between Electrodes and Condition could only be related to the detection of a change in the deviant condition. For each analysis, the random variable was the blocks recorded in each experiment. In these analyses, main effects are not interpretable because of the voltage inversion between the selected Electrodes; therefore, only interactions between Electrodes and the other factors of the analyses were examined.

\subsection{Results}

All types of sounds (tones, vowels, and CV syllables) induced, right-lateralized evoked responses made up of a frontal positivity synchronous with a right temporal negativity (Fig. 2). The global field power analysis showed that L.G.'s responses peaked at $386 \mathrm{~ms}$ post-
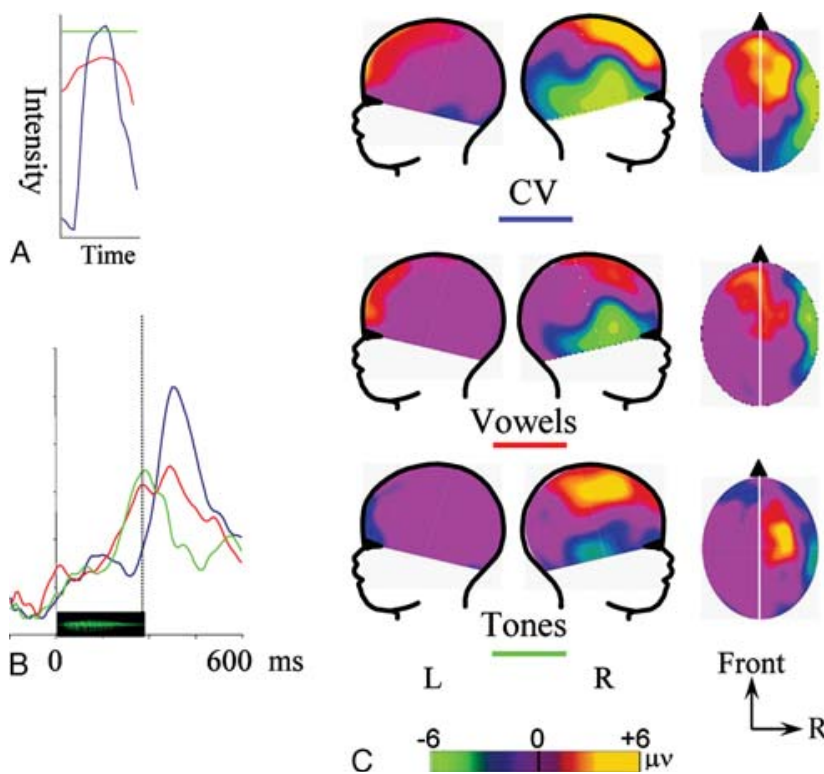

Fig. 2. Experiment 1: (A) intensity envelope for the three types of stimuli; (B) global field power of the grand average for the first stimulus of the trials; and $(\mathrm{C})$ voltage cartographies at the first maximum of the global field power for the three types of stimuli: tones, vowels, and $\mathrm{CV}$ syllables. Notice the asymmetry of the response with weaker voltage above the left pathological side. onset for CV syllables, 298 and $378 \mathrm{~ms}$ post-onset for vowels and at $290 \mathrm{~ms}$ post-onset for tones (Fig. 2). The $90 \mathrm{~ms}$ delay in the latency of the peak maximum for $\mathrm{CV}$ syllables as compared to the tones and vowel peak corresponds to the $85 \mathrm{~ms}$ duration of the prevoiced and voiced parts that delay the intensity maximum.

\subsubsection{Habituation analysis}

As with normal infants, repetition of the same stimulus led to a decrease in the amplitude of the evoked response. The ANOVA performed on the amplitude of the peaks for each of the first 3 stimuli in the trials, with Electrodes, Number of stimuli (1, 2, and 3), and Hemisphere as within-block factors and Stimulus Type (Tones, Vowels, and Stop consonants) as a betweenblocks factor, confirmed the significant decrease in amplitude of the ERP with stimulus repetition (Stimulus number by Electrodes, $F(2,42)=27.1, p<.0001)$. This decrease was more prominent over the right than the left hemisphere (Stimulus number by Electrodes by Hemisphere $F(2,42)=29.1, p<.0001)$. These two comparisons were significant when restricted to each type of stimulus (all $p s<.05$ ). The significant interaction between Hemisphere and Electrodes $(F(1,21)=80.7$, $p<.0001)$ confirmed the strong right-lateralization of the ERP in L.G.; this right-lateralization was present for each type of stimulus (all $p$ s $<.05$ ).

\subsubsection{Mismatch analysis}

The introduction of a deviant stimulus induced an increase of amplitude for all types of stimuli as compared to the standard condition. The mismatch response began at around $306 \mathrm{~ms}$ for the tones and the vowels, and later

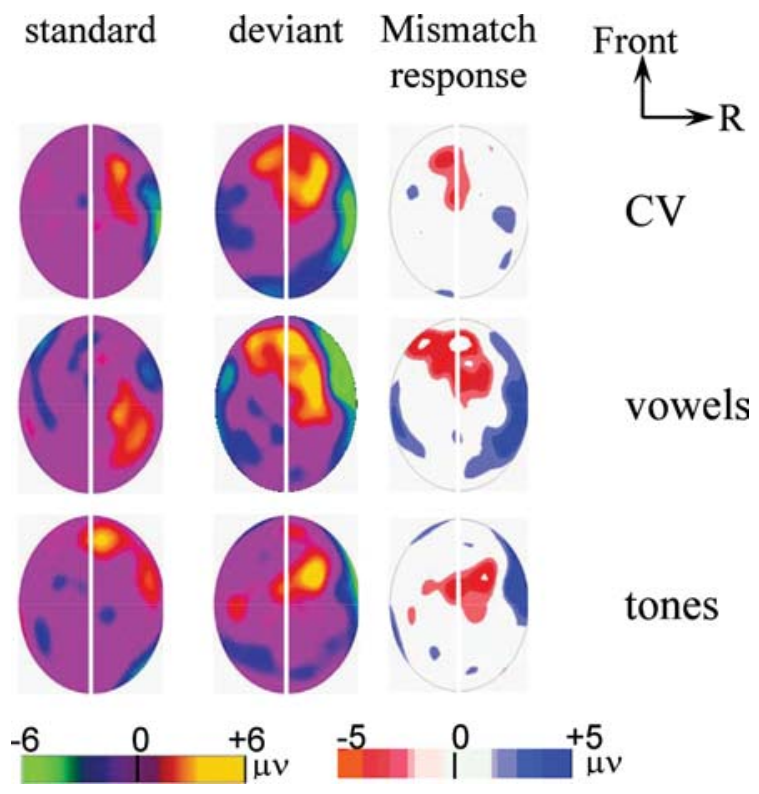

Fig. 3. Experiment1: Voltage cartographies for the deviant and standard condition at $400 \mathrm{~ms}$ post onset of the 4 th stimulus and mismatch responses (deviant-standard) for the three types of stimuli. 
for the CV syllables, around $346 \mathrm{~ms}$ after the onset of the deviant stimulus. It lasted longer for tones and vowels than for CV syllables (266 ms vs $144 \mathrm{~ms}$ ). Its topography partially overlapped across types of stimuli although the anterior positivity was more central and the negativity extended less posteriorly for the tones than for linguistic stimuli: vowels and CV syllables (Fig. 3).

To analyze the discrimination (or mismatch) response, four non-overlapping clusters of 5 or 6 electrodes were selected around the poles of the mismatch responses for tones (central and lateral) and for syllables (frontal and occipito-temporal). Voltage was averaged over a $100 \mathrm{~ms}$ temporal window, between 350 and $450 \mathrm{~ms}$ post-onset (in this window, the mismatch response was present for all types of stimuli). It was then entered in an ANOVA with Electrodes (4 sites), Hemisphere (left and right), and Condition (standard and deviant) as within-block factors and Stimulus Type as a between-blocks factor. As in the habituation analysis, the interaction Hemisphere $\times$ Electrodes was significant $(F(3,63)=21.76, p<.0001)$, with higher voltage over the right hemisphere. This asymmetry was more marked for the CV syllables than for the other stimuli (Table 1) generating a significant triple interaction Hemisphere $\times$ Electrodes $\times$ Type of stimuli $(F(6,63)=2.77$, $p=.02)$. The mismatch response was significant when all stimuli were collapsed (Electrodes $\times$ Condition: $F(3,63)=9.08, p<.0001)$ and when the comparisons were restricted to each type of stimulus ( $p \mathrm{~s}<.05$, Table 1). There was no significant left-right asymmetry in the mismatch response (Condition $\times$ Hemisphere $\times$ Electrodes: $F(3,63)=1.52, p=.22$ and Condition $\times$ Hemisphere $\times$ Electrodes $\times$ Type of stimulus: $F(6,63)<1$ ). When analyses were restricted to each type of stimulus, only the mismatch response for the tones was significantly predominant over the right hemisphere relative to the left (Table 1). The absence of asymmetry in the two other conditions is due to the topography of the positivity which is situated toward the middle, and expanding to the left frontal area.

\subsection{Discussion}

With L.G., as with normal infants (Dehaene-Lambertz \& Dehaene, 1994), repetition of an identical stimulus induced a decrease in the amplitude of the evoked response while the introduction of a deviant stimulus generated a mismatch response. This functional pattern was present for each type of stimulus: tones, vowels, and CV syllables, as demonstrated by the significant Electrodes $\times$ Condition interaction present for each type of stimulus. The mismatch response was longer for tones and vowels than for consonants. This is probably due to the brief duration of the change in the CV syllables. /ba/ and /ga/ differ only during the $40 \mathrm{~ms}$ of the formant transition, whereas tones and vowels differ during the whole duration of the stimulus. Similarly the differences in the latencies of the ERP are related to the acoustical characteristics of the stimuli. For tones and vowels, energy is immediately at a maximum while with the CV syllables, it is delayed until the vowel onset (see the stimuli intensity envelope in Fig. 2A). All these elements demonstrate that despite L.G.'s sizeable left hemisphere lesion, her remaining functional brain tissue was able to process auditory stimuli efficiently and differentially, even when they were as brief and fast as a place of articulation contrast.

However, if L.G. was able to discriminate two consonants $/ \mathrm{b} /$ and $/ \mathrm{g} /$, this does not prove that the discrimination response was based on a linguistic network. As a matter of fact, an acoustic processor of fast transition formants allows for the discrimination of two acoustic exemplars such as $/ \mathrm{b} /$ and $/ \mathrm{g} /$ whereas it might have trouble considering that several exemplars of $/ b /$ belong to the same category and opposing them to exemplars of another category, such as /g/. But human listeners have no difficulty in identifying a particular syllable when its acoustic characteristics undergo considerable variation due to the speaker's vocal tract size and shape, speech register, speech rate, environmental noise, etc. This capacity to extract phonetic categories in

Table 1

Statistical analyses of the mismatch responses in Experiment 1

\begin{tabular}{|c|c|c|c|c|}
\hline Comparisons & Stimuli collapsed & Tones & Vowels & Consonants \\
\hline E.H & $F(3,63)=21.8^{* * *}$ & $F(3,15)=3.7^{*}$ & $F(3,15)=3.1$ & $F(3,33)=21.6^{* * *}$ \\
\hline E.C & $F(3,63)=9.1^{* * *}$ & $F(3,15)=3.3^{*}$ & $F(3,15)=4.7^{*}$ & $F(3,33)=3.1^{*}$ \\
\hline E.C.H & $F(3,63)=1.5$ & $F(3,15)=3.5^{*}$ & $F(3,15)<1$ & $F(3,33)<1$ \\
\hline E.C over the RH & $F(3,63)=8.5^{* * *}$ & $F(3,15)=4.6^{*}$ & $F(3,15)=3.6^{*}$ & $F(3,33)=2.6$ \\
\hline E.C over the LH & $F(3,63)=5.8^{* *}$ & $F(3,15)=1.9$ & $F(3,15)=2.9$ & $F(3,33)=2.7$ \\
\hline $\mathrm{C}$ (at the frontal site) & $F(1,21)=9.3^{* *}$ & $F(1,5)<1$ & $F(1,5)=3.9$ & $F(1,11)=5.2^{*}$ \\
\hline $\mathrm{C}$ (at the central site) & $F(1,21)=7.6^{*}$ & $F(1,5)=8.3^{*}$ & $F(1,5)=1.8$ & $F(1,11)=1.8$ \\
\hline $\mathrm{C}$ (at the temporo-occipital site) & $F(1,21)=9.8^{* *}$ & $F(1,5)=1.5$ & $F(1,5)=5.1$ & $F(1,11)=4.8$ \\
\hline $\mathrm{C}$ (at the temporal site) & $F(1,21)=8.9^{* *}$ & $F(1,5)=6.9^{*}$ & $F(1,5)=8.2^{*}$ & $F(1,11)=1.3$ \\
\hline
\end{tabular}

E, electrodes; H, hemisphere; R, right; L, left, $\mathrm{C}$, condition.

${ }^{*} p<.05$.
${ }^{* *} p<.01$.
${ }^{* * *} p<.001$. 
the presence of irrelevant acoustical variations is essential to speech comprehension, and normalization is one of the arguments used to postulate specific networks dedicated to phoneme coding in the human brain ( $\mathrm{Li}-$ berman, 1996). In Experiment 2, we thus explored how L.G.'s discrimination response was affected by irrelevant speaker variation.

\section{Experiment 2: Normalization in phoneme perception}

We used the same experimental paradigm as in Experiment 1 . We again tested a place of articulation contrast because there are no simple acoustical cues that can be computed across speakers to identify a phonetic category along the place of articulation dimension (Stevens $\&$ Blumstein, 1981) and we already had recordings of the responses to this contrast in normal sleeping neonates that allowed us to compare L.G.'s responses to theirs.

\subsection{Method}

\subsubsection{Stimuli}

Two syllables $/ \mathrm{pa} /$ and $/ \mathrm{ta} /$ produced by 4 female speakers were used. The average root mean square of the syllable waveforms was equalized and the syllables were presented at $78 \mathrm{~dB}$ SPL. The fundamental frequency for each speaker was $205,183,177$, and $184 \mathrm{kHz}$ (Praat 3.9.3 software www.praat.org). Syllable intonation and duration varied across speakers $(219,263,250$, $229 \mathrm{~ms}$ for $/ \mathrm{ta} /$ and $227,242,267$, and 257 for $/ \mathrm{pa} /$ ). The syllables were presented in pairs to naive adults who were able to perceive a change of speaker in $92 \%$ of the similar phonetic pairs thus indicating that the acoustical variations between the different utterances of each syllable were indeed perceptible.

\subsubsection{Procedure}

The procedure was similar to the one used in Experiment 1 . ERPs were recorded in 8 sessions of about $30 \mathrm{mn}$, from 5 to 6 weeks of life. Each session was made up of two blocks of 120 trials. Sixteen blocks were thus obtained in this experiment. The standard was kept constant within a block. In each block, 4 types of trials were presented: standard (A A A A) vs deviant (A A A B) and "same speaker" vs "different speakers". In "same speaker" trials, the same physical syllable was repeated three times followed either by itself in standard trials, or by the deviant syllable produced by the same speaker in deviant trials (speaker 1). In "different speakers" trials, each syllable was produced by a different speaker. Order of speakers was randomly constituted for each trial with no repetition of speaker within the same trial, the last syllable always being produced by speaker 1 . In standard trials, the phonetic category was maintained whereas in deviant trials there was a change of phoneme for the last syllable of the trial. After rejection, an average of 27.6 trials was kept in each block and each condition.

\subsubsection{Control subjects}

Sixteen healthy sleeping neonates (mean age 3.5 days) were tested with the same paradigm. The results of this experiment have been reported elsewhere (DehaeneLambertz \& Pena, 2001).

\subsection{Results}

Syllables elicited the same right-lateralized ERP as that described in Experiment 1, peaking at $302 \mathrm{~ms}$ postonset for both speaker conditions (Fig. 4). The shorter latency of the peak in Experiment 2 as compared to the responses to /ba/ /ga/ in Experiment 1 ( $84 \mathrm{~ms}$ earlier) is related to the shorter duration of the voiceless consonants $/ \mathrm{pa} / / \mathrm{ta} /$ relative to the voiced consonants /ba//ga/.

\subsubsection{Habituation analysis}

As in Experiment 1, the voltage was higher above the right than the left hemisphere (Electrode $\times$ Hemisphere $F(1,15)=45.98, p<0.001)$ and the decrease of the ERP amplitude with repetition was significant (Stimulus number $\times$ Electrodes: $F(2,30)=37.5, p<.0001)$, and greater over the right than the left hemisphere (Stimulus number $\times$ Electrodes $\times$ Hemisphere: $\quad F(2,30)=44.67$, $p<.0001)$, with no significant interaction with type of trials $(F \mathrm{~s}<1)$. Habituation was present in Samespeaker trials $(F(2,30)=39.76, p<.0001)$ as well as in Different-speaker trials $(F(2,30)=18.48, p<.0001)$.

\subsubsection{Mismatch response}

A mismatch response was present for both the samespeaker and different-speakers' trials, beginning at $238 \mathrm{~ms}$ post-onset and lasting about $150 \mathrm{~ms}$. Its topography was similar to the topography of the mismatch response for CV syllables in Experiment 1 (Fig. 5). The frontal positivity extended more to the left in the different-speakers condition than in the same-speaker condition.

The same clusters of electrodes as in Experiment 1 were used for the statistical analyses. An Anova was computed on the voltage averaged on a $100 \mathrm{~ms}$ time-window, beginning at $250 \mathrm{~ms}$ post-onset, with the same within-block factors as in Experiment 1 (Electrodes, Hemisphere, Condition) plus the between-block factor Type of trials (Same-speaker vs Different-speakers). The mismatch response was significant (Electrode $\times$ Condition: $F(3$, $45)=5.63, p=.002)$ and similar for both types of trials (Electrode $\times$ Condition $\times$ Type of trials: $F(3,45)=1.04$, $p=.38)$. The triple interaction Electrode $\times$ Condition $\times$ Hemisphere was significant only for the samespeaker condition $(F(3,45)=3.82, p=.017)$ while only the Electrode $\times$ Condition interaction was significant for the Different-speakers condition $(F(3,45)=5.26$, 


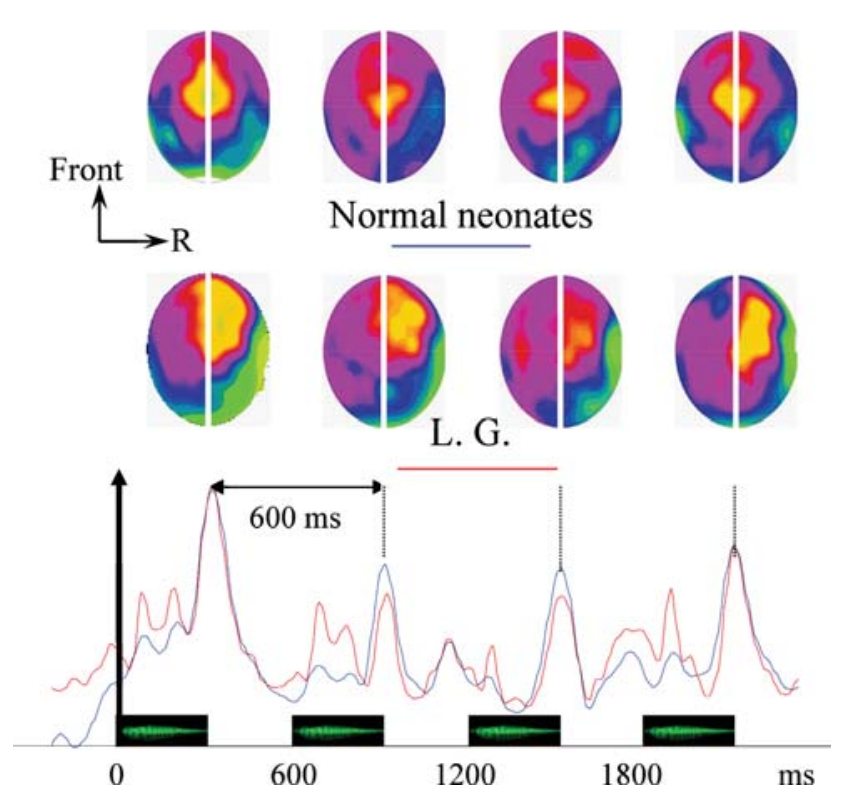

Fig. 4. Experiment 2: Global field power (last row) of the grand average during an entire trial and voltage cartographies at the maximum of the global field power for each syllable used with L.G. (second row) and normal neonates (first row). All conditions are collapsed. The latencies of the peaks are similar although the response is more rightlateralized in L.G. than for normal neonates. Repetition of similar stimuli induces an habituation of the ERP as demonstrated by the decrease in amplitude of the global field power and of the voltage on the cartographies.

$p=.003$ ) because of the extension of the frontal positivity on the left side in this condition. As with the CV syllables in Experiment 1, the mismatch response was significantly present over the frontal and temporo-occipital sites (Table 2).

\section{Experiment 2: Comparison with healthy sleeping neonates}

A group of 16 healthy neonates was also tested under exactly the same conditions as those used in Experiment 2 (Dehaene-Lambertz \& Pena, 2001). The latency of the components were similar for L.G. and for the neonates with a maximum global field power at $302 \mathrm{~ms}$ post onset in the two sets of data although the voltage cartographies were more asymmetric toward the right for L.G. than for the normal neonates (Fig. 5).

To compare the discrimination responses, we performed an ANOVA with the same factors as above plus pathology as a between-subjects variable (16 blocks in L.G. vs 16 neonates). The Electrode $\times$ Condition interaction, indicating a mismatch response, was significant $(F(3,90)=8.09, p=.001)$ and did not interact with Pathology $(F(3,90)=1.67, p=.18)$. The voltage asymmetry was reversed in L.G. relative to the normal neonates, as indicated by the significant Electrode $\times$ Hemisphere $\times$ Pathology triple interaction
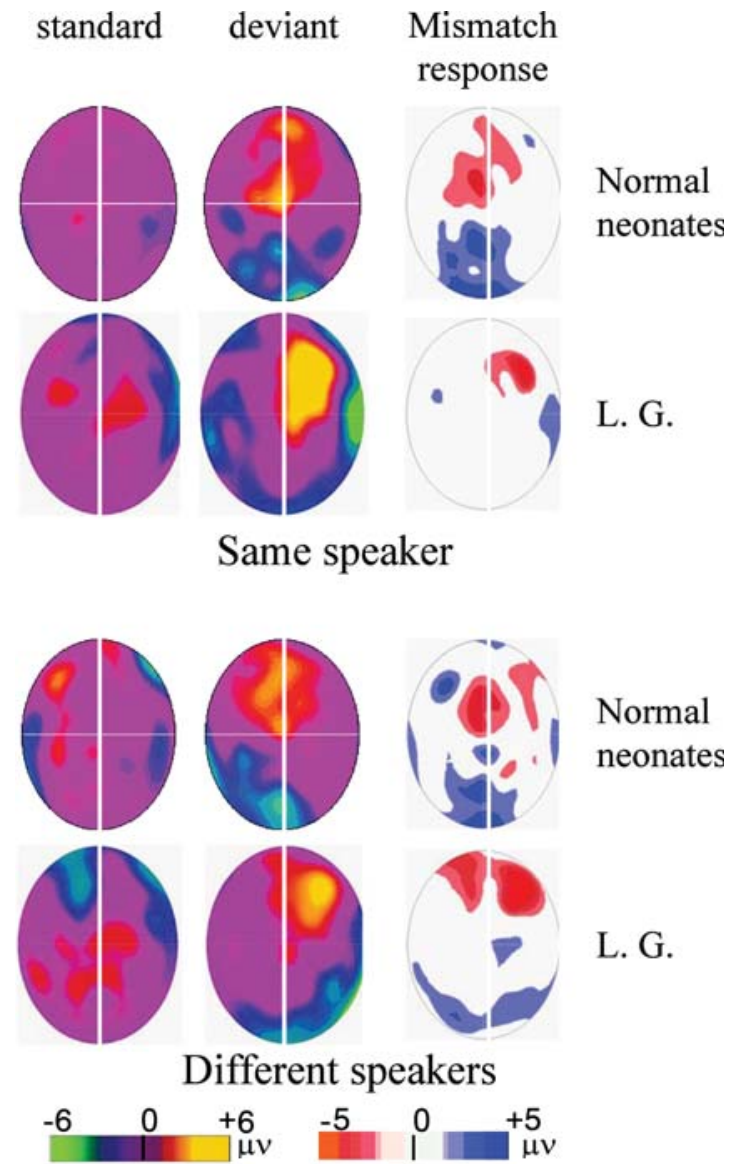

Fig. 5. Experiment 2: Voltage cartographies for the standard and deviant condition at $300 \mathrm{~ms}$ post onset of the deviant stimulus and mismatch responses in the "same speaker" and in the "different speaker" trials, in L.G. and for normal neonates. The response is more rightlateralized in L.G than for normal neonates. Despite her left lesion, L.G. is able to discriminate a change of consonant even when the syllables are produced by different speakers. This indicates that her right hemisphere is able to extract the relevant phonetic information despite irrelevant acoustic variation.

$(F(3,90)=15.62, p<.0001)$. Over the left hemisphere, there was a significant Electrode $\times$ Pathology interaction $(F(3,90)=6.28, p<.001)$ due to the overall low voltage over this hemisphere for L.G. in comparison with normal infants, while the same interaction Electrodes $\times$ Pathology was of much less amplitude over the right hemisphere and due to higher voltage in L.G. than in normal neonates $(F(3,90)=3.49, p<.02)$.

\subsection{Discussion}

L.G., a full-term neonate with a correctly supervised and unproblematic pregnancy, presented a wide lesion in the left hemisphere as a result of a difficult delivery owing to a macrosomic neonate. The lesion had destroyed the entire left sylvian territory. In adults, such a large left lesion would produce aphasia with a severe deficit in phoneme discrimination. We therefore tested 
Table 2

Statistical analyses of the mismatch responses in Experiment 2

\begin{tabular}{llll}
\hline Comparisons & Stimuli collapsed & Same speaker & Different speakers \\
\hline E.H & $F(3,45)=19.6^{* * *}$ & $F(3,45)=9.6^{* * *}$ & $F(3,45)=10.2^{* * *}$ \\
E.C & $F(3,45)=5.7^{* *}$ & $F(3,45)=1.5$ & $F(3,45)=5.3^{* *}$ \\
E.C.H & $F(3,45)=2.0$ & $F(3,45)=3.8^{*}$ & $F(3,45)<1$ \\
E.C over the RH & $F(3,45)=5.6^{* *}$ & $F(3,45)=3.2^{*}$ & $F(3,45)=3.4^{*}$ \\
E.C over the LH & $F(3,45)=3.1^{*}$ & $F(3,45)<1$ & $F(3,45)=5.9^{* *}$ \\
C (at the frontal site) & $F(1,15)=7.3^{*}$ & $F(1,15)=1.6$ & $F(1,15)=7.1^{*}$ \\
C (at the central site) & $F(1,15)<1$ & $F(1,15)=1.0$ & $F(1,15)<1$ \\
C (at the temporo-occipital site) & $F(1,15)=8.7^{* *}$ & $F(1,15)=2.8$ & $F(1,15)=6.7^{*}$ \\
C (at the temporal site) & $F(1,15)=2.2^{*}$ & $<1$ & $F(1,15)=2.5$ \\
\hline
\end{tabular}

E, electrodes; $\mathrm{H}$, hemisphere; $\mathrm{R}$, right; $\mathrm{L}$, left; $\mathrm{C}$, condition.

${ }^{*} p<.05$.

${ }^{* *} p<.01$.

$p<.001$

L.G. a few weeks after the lesion to determine the auditory capacities in the remaining right hemisphere, in particular the capacity to process phonemes. Both the initial MRI (Fig. 1) and the one performed 6 months later demonstrated the destruction of most of the left hemisphere and particularly of the peri-sylvian regions that are involved in the processing of auditory stimuli. In consequence, the auditory ERP in L.G. was strongly lateralized, yielding abnormal right-lateralized topographies compared to those of healthy neonates.

Despite her severe lesion, L.G. was able to process auditory stimuli remarkably efficiently, in a manner similar to healthy infants. The latencies of the evoked responses were not delayed compared to those of normal neonates (Fig. 4). Repetition of similar stimuli, either physically identical or different but belonging to the same phonetic category, induced a decrease in the amplitude of the evoked response. Finally, the introduction of a deviant stimulus generated a mismatch response similar to the one described for normal infants. This functional pattern demonstrates that despite the lesion, L.G.'s brain was able to code the similarity of auditory stimuli and react to a change, just as normal infants do (Dehaene-Lambertz \& Dehaene, 1994). Several authors have attributed the capacity for fine temporal analysis to the left hemisphere (Tallal \& Piercy, 1974; Zatorre \& Belin, 2001). L.G.'s remaining right auditory cortex was able to respond differentially to temporal differences in auditory stimuli. Her evoked responses were affected by the dynamics of the acoustic signal, as is the case with adults (Sharma \& Dorman, 1999) or normal infants (Dehaene-Lambertz, 2000). Moreover, the consonant discrimination in Experiments 1 and 2 relied on the perception of a fast temporal change occurring within the $20-40 \mathrm{~ms}$ period during which the consonants differed. The presence of a mismatch response in both cases indicates that the right hemisphere, at least in this neonate, was capable of fine temporal analyses. This pattern of results thus suggests that the asymmetry observed in adults is probably not due to an initial inability of the right hemisphere to perform these analyses; rather, it may be a functional consequence of development.

Experiment 2 demonstrates that L.G. was not only able to process fast transitions but also to compute phoneme representations independently of the acoustical variations present in the stimuli. Similar mismatch responses were observed in Experiments 1 and 2 with the same topography above the frontal and temporo-occipital sites. Moreover, this discrimination response was unaffected by irrelevant acoustical variations in the different-speakers condition. In the experimental design used here, we compared ERPs to the same syllable at the same 4th position in standard and deviant trials. Thus any significant difference between these conditions can only be related to the computation of how dissimilar the incoming stimulus was relative to the previous stimuli. In the Different-speakers trials, although timbre, pitch, duration, and intonation varied across the three first syllables, a change of phonetic category in the 4th position in deviant trials was perceived as more dissimilar than another change of these acoustic characteristics in standard trials. This generated a significant difference between the standard and deviant conditions, demonstrating that the phoneme category was computed despite the acoustical variations. Because no simple acoustical cues can be computed across speakers to identify phonetic category (Stevens \& Blumstein, 1981), the ability to normalize has been described as one of the main arguments to suggest the presence of specific networks dedicated to phoneme coding in the human brain (Liberman, 1996). It thus seems that L.G.'s right hemisphere possesses the capacity to normalize across irrelevant variation and perceive phonetic categories. Although there was no interaction between the mismatch response and the type of trials in Experiment 2, the mismatch response had a greater amplitude in the Different-speakers condition than in the Same-speaker condition. This characteristic has also been found for normal neonates (Dehaene-Lambertz \& Pena, 2001). There is no clear explanation for this effect except 
that several exemplars of a category may elicit a more abstract and thus better prototypical representation of the phonetic category than a single exemplar.

In adults, a phonetic deficit after a left hemispheric lesion has mainly been shown with behavioral studies. Here, we present electrophysiological responses instead. No behavioral data were gathered for L.G. because no behavioral paradigm at this age is sufficiently reliable to assess the discrimination capacities of an individual subject (for instance, the non-nutritive sucking paradigm typically requires testing groups of 40 infants, see for example Bertoncini et al., 1989). Furthermore, L.G.'s vigilance was affected by the recent and severe brain lesion. She was hard to wake up or keep attentive, discouraging any attempt to obtain behavioral data in any of the behavioral paradigms generally used with neonates. Several studies have nevertheless demonstrated a good correlation between the presence and duration of the mismatch response, and the subjects' performance on auditory discrimination tasks, either in patients (Aaltonen, Tuomainen, Laine, \& Niemi, 1993; Csepe, Osman-Sagi, Molnar, \& Gosy, 2001), children with learning problems (Kraus et al., 1996) or normal adults (Tiitinen, May, Reinikainen, \& Näätänen, 1994). In experiments where adults are trained to perceive a change between stimuli that they do not perceive initially, a change in the mismatch response duration precedes and is correlated with an improvement in discrimination performance (Kraus et al., 1995; Tremblay, Kraus, \& McGee, 1998). Even in the absence of behavioral data, the presence of a mismatch response in L.G. indicates at the very least that a neural network was able to discriminate a phonetic change while she was inattentive and asleep. Similar results were found with healthy neonates, for whom mismatch responses were also recorded when they were asleep (Cheour et al., 2002b; Dehaene-Lambertz \& Pena, 2001).

Besides normalization, categorical perception is another property typical of phonetic processing. In earlier work, we demonstrated that the mismatch response to a phoneme change in infants possesses these two properties, suggesting that a phonetic network is effective very early in life (Dehaene-Lambertz, 1997; Dehaene-Lambertz \& Pena, 2001). However, with normal infants we did not find stronger left activation for a linguistic change as compared to a non-linguistic change, such as a timbre (Dehaene-Lambertz, 2000). For both linguistic and nonlinguistic stimuli, the ERPs were largely bilateral, with significantly higher voltages recorded above the left hemisphere. We concluded from these results that the lateralization pattern during the first months of life was different than the one for adults, with infants demonstrating greater left hemisphere responses to any type of sound. It is possible that the higher activation of the left auditory networks, within a speech environment, contributes to the development of the adult's lateralization pattern by inhibiting the opposite right cortices through the corpus callosum. Reggia, Goodall, and Skuro (1998) proposed a plausible computational model for this phenomenon and demonstrated spontaneous lateralization of the capacity to generate a sequence of phonemes in a pair of connected "hemispheric" regions. Lateralization was found on the side that was larger and/or demonstrated higher reactivity, and/or greater synaptic plasticity, and was, therefore the consequence of "a race to learn." The experimental evidence obtained from L.G and from healthy infants suggests that the neonate brain possesses neuronal networks capable of processing phonemes in much the same way as adults do. Nativists would of course expect this, but given that the balance between the left and right hemispheres is not the same in infants and adults, the strong asymmetry observed in adults would appear to be the result of maturational and/ or exogenous factors.

Finally, we would like to draw attention to a methodological point. Hitherto, problems inherent to behavioral experiments prevented any cognitive study of an individual subject at an early age. Functional brain imaging techniques, such as event-related potentials, have changed this and we can now study young and non-cooperative subjects with none of the problems that were encountered with the behavioral research. In the work we report here, we were able to obtain reliable data from a single infant, by recording over multiple sessions. Even though it is, in principle, possible to use the same multiple-sessions strategy in behavioral paradigms, we do not know of any paper that evaluated the cognitive capacities of an individual subject participating in a behavioral experiment (especially at this early age when only non-nutritive sucking is available). To the best of our knowledge, we have demonstrated for the first time that the ERP paradigm as we use it allows experimenters to gather reliable data on the cognitive capacities of a single brain-damaged infant.

\section{Acknowledgments}

This study was supported by grants from the Ministère Français de la Santé et de la Recherche PHRC 1995 No. AOM95011, Groupement d'Intérêt Scientifique Sciences de la Cognition No. PO 9004, Contrat

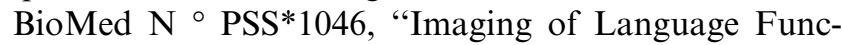
tions in the Brain," 1999-2001, ACI Blanche 1999 "Plasticiténeuronale et acquisition du langage," and a Mc Donnell Foundation grant to G.D.L.

\section{References}

Aaltonen, O., Tuomainen, J., Laine, M., \& Niemi, P. (1993). Cortical differences in tonal versus vowel processing as revealed by an ERP 
component called mismatch negativity (MMN). Brain and Language, 44, 139-152.

Ahoniska, J., Cantell, M., Tolvanen, A., \& Lyytinen, H. (1993). Speech perception and brain laterality: The effect of ear advantage on auditory event-related potential. Brain and Language, 45, 127-146.

Aram, D. M., \& Ekelman, B. L. (1986). Spoken syntax in children with acquired unilateral hemisphere lesions. Brain and Language, 27, 75100.

Aram, D. M., Meyers, S. C., \& Ekelman, B. L. (1990). Fluency of conversational speech in children with unilateral brain lesions. Brain and Language, 38, 105-121.

Bates, E., \& Roe, K. (2001). Language development in children with unilateral brain injury. In C. Nelson, \& M. Luciana (Eds.), Handbook of developmental cognitive neurocsience (pp. 281-307). Cambridge, MA: MIT Press.

Bates, E., Vicari, S., \& Trauner, D. (1999). Neural mediation of language development: Perspectives from lesion studies of infants and children. In H. Tager-Flusberg (Ed.), Neurodevelopmental disorders (pp. 533-581). Cambridge, MA: MIT Press.

Bertoncini, J., Morais, J., Bijeljac-Babic, R., McAdams, S., Peretz, I., \& Mehler, J. (1989). Dichotic perception and laterality in neonates. Brain and Language, 37, 591-605.

Best, C. T. (1988). The emergence of cerebral asymmetries in early human development: A literature review and a neuroembryological model. In D. Molfese, \& S. J. Segalowitz (Eds.), Brain lateralization in children. New York: The Guilford Press.

Best, C. T., Hoffman, H., \& Glanville, B. B. (1982). Development of infant ear asymmetries for speech and music. Perception \& Psychophysics, 31, 75-85.

Binder, J. R., Frost, J. A., Hammeke, T. A., Bellgowan, P. S., Springer, J. A., Kaufman, J. N., et al. (2000). Human temporal lobe activation by speech and non speech sounds. Cerebral Cortex, 10(5), 512-528.

Blumstein, S. E., Baker, E., \& Goodglass, H. (1977). Phonological factors in auditory comprehension in aphasia. Neuropsychologia, $15,19-30$.

Boatman, D., Hall, C., Goldstein, M. H., Lesser, R., \& Gordon, B. (1997). Neuroperceptual differences in consonant and vowel discrimination: As revealed by direct cortical electrical interference. Cortex, 33, 83-98.

Celsis, P., Boulanouar, K., Doyon, B., Ranjeva, J. P., Berry, I., \& Chollet, F. (1999). Differential fMRI responses in the left posterior superior temporal gyrus and left supramargynal gyrus to habituation and change detection in syllables and tones. Neuroimage, 9, 135-144.

Cheour, M., Ceponiene, R., Leppanen, P., Alho, K., Kujala, T., Renlund, M., et al. (2002a). The auditory sensory memory trace decays rapidly in newborns. Scandinavian Journal of Psychology, 43(1), 33-39.

Cheour, M., Martynova, O., Naatanen, R., Erkkola, R., Sillanpaa, M., Kero, P., et al. (2002b). Speech sounds learned by sleeping newborns. Nature, 415(6872), 599-600.

Chiron, C., Jambaque, I., Nabbout, R., Lounes, R., Syrota, A., \& Dulac, O. (1997). The right brain hemisphere is dominant in human infants. Brain, 120, 1057-1065.

Chobor, K. L., \& Brown, J. W. (1987). Phoneme and timbre monitoring in left and right cerebrovascular accident patient. Brain and Language, 30, 278-284.

Corballis, M. C. (1991). The lopsided ape. Evolution of the generative mind. Oxford: Oxford University Press.

Csepe, V., Osman-Sagi, J., Molnar, M., \& Gosy, M. (2001). Impaired speech perception in aphasic patients: Event-related potential and neuropsychological assessment. Neuropsychologia, 39(11), 11941208.

de Haan, M., \& Nelson, C. A. (1999). Brain activity differentiates face and object processing in 6-month-old infants. Developmental Psychology, 35(4), 1113-1121.
Dehaene, S., Dupoux, E., Mehler, J., Cohen, L., Paulesu, E., Perani, D., et al. (1997). Anatomical variability in the cortical representation of first and second languages. Neuroreport, 8, 38093815.

Dehaene-Lambertz, G. (1997). Electrophysiological correlates of categorical phoneme perception in adults. Neuroreport, 8(4), 919924.

Dehaene-Lambertz, G. (2000). Cerebral specialization for speech and non-speech stimuli in infants. Journal of Cognitive Neuroscience, 12(3), 449-460.

Dehaene-Lambertz, G., \& Baillet, S. (1998). A phonological representation in the infant brain. Neuroreport, 9, 1885-1888.

Dehaene-Lambertz, G., \& Dehaene, S. (1994). Speed and cerebral correlates of syllable discrimination in infants. Nature, 370, 292 295.

Dehaene-Lambertz, G., Dehaene, S., \& Hertz-Pannier, L. (2002). Functional neuroimaging of speech perception in infants. Science, 298, 2013-2015.

Dehaene-Lambertz, G., \& Pena, M. (2001). Electrophysiological evidence for automatic phonetic processing in neonates. Neuroreport, 12, 3155-3158.

Eimas, P. D., \& Miller, J. L. (1992). Organization in the perception of speech by young infants. Psychological Science, 3, 340-345.

Eimas, P. D., Siqueland, E. R., Jusczyk, P. W., \& Vigorito, J. (1971). Speech perception in infants. Science, 171, 303-306.

Fischer, C., Morlet, D., Bouchet, P., Luaute, J., Jourdan, C., \& Salord, F. (1999). Mismatch negativity and late auditory evoked potentials in comatose patients. Clinical Neurophysiology, 110, 16011610 .

Glanville, B. B., Best, C. T., \& Levenson, R. (1977). A cardiac measure of cerebral asymmetries in infant auditory perception. Developmental Psychology, 13, 54-59.

Imaizumi, S., Mori, K., Kiritani, S., Kawashima, R., Sugiura, M., Fukuda, H., et al. (1997). Vocal identification of speaker and emotion activates different brain regions. Neuroreport, 8(12), 28092812.

Jacquemot, C., Pallier, C., LeBihan, D., Dehaene, S., \& Dupoux, E. (submittted). Cerebral bases of phonological illusions: A crosslinguistic brain imaging study.

Kim, K. H. S., Relkin, N. R., Lee, K.-M., \& Hirsch, J. (1997). Distinct cortical areas associated with native and second languages. Nature, 338, 171-174.

Kraus, N., McGee, T., Carrell, T. D., King, C., Tremblay, K., \& Nicol, T. (1995). Central auditory system plasticity with speech discrimination training. Journal of Cognitive Neuroscience, 7, 2532.

Kraus, N., McGee, T. J., Carrel, T. D., Zecker, S. G., Nicol, T. G., \& Koch, D. B. (1996). Auditory neurophysiologic responses and discrimination deficits in children with learning problems. Science, 273, 971-973.

Kuhl, P. K. (1983). Perception of auditory equivalence classes for speech in early infancy. Infant Behavior and Development, 6, 263285.

Lenneberg, E. H. (1967). Biological foundations of language. New York: Wiley.

Liberman, A. M. (1996). Speech: A special code. Cambridge, MA: Bradford Books/MIT Press.

Mehler, J., \& Dupoux, E. (1994). What infants know. Cambridge, MA: Basil Blackwell.

Miceli, G., Caltagirone, C., Gainotti, G., \& Payer-Rigo, P. (1978). Discrimination of voice versus place contrasts in aphasia. Brain and Language, 2, 434-450.

Molfese, D. L., \& Burger-Judisch, L. M. (1991). Dynamic temporalspatial allocation of resources in the human brain. An alternative to the static view of hemispheric differences. In F. L. Kitterle (Ed.), Cerebral laterality: Theory and research (pp. 71-102). Hillsdale, NJ: Erlbaum. 
Molfese, D. L., \& Molfese, V. J. (1979). Hemisphere and stimulus differences as reflected in the cortical response of newborn infants to speech stimuli. Developmental Psychology, 15, 501-511.

Näätänen, R., Lehtokovski, A., Lennes, M., Cheour, M., Huotilainen, M., Iivonen, A., et al. (1997). Language-specific phoneme representations revealed by electric and magnetic brain responses. Nature, 385, 432-434.

Nielsen-Bohlman, L., Knight, R. T., Woods, D. L., \& Woodward, K. (1991). Differential auditory processing continues during sleep. Electroencephalography and Clinical Neurophysiology, 79(4), 281290.

Novak, G. P., Kurtzberg, D., Kreuzer, J. A., \& Vaughan, J. H. G. (1989). Cortical responses to speech sounds ant their formants in normal infants: Maturational sequences and spatiotemporal analysis. Electroencephalography and Clinical Neurophysiology, 73, 295305 .

O'Leary, D. S., Andreasen, N. C., Hurtig, R. R., Hichiwa, R. D., Watkins, G. L., Ponto, L. L. B., et al. (1996). A positron emission tomography study of binaurally and dichotically presented stimuli: Effects of level of language and directed attention. Brain and Language, 53, 20-39.

Pallier, C., Dupoux, E., \& Jeannin, X. (1997). EXPE: an expandable programming language for on-line psychological experiments. Behavior Research Methods, Instruments, \& Computers, 29, 322 327.

Pena, M., Maki, A., Kovacic, D., Dehaene-Lambertz, G., Bouquet, F., \& Koizumi, H., et al. (in press). Sounds and silence: An optical topography study of language recognition at birth. PNAS.

Pinker, S. (1994). The language instinct: How the mind creates language. London: Penguin.

Reggia, J. A., Goodall, S., \& Skuro, Y. (1998). Computational studies of lateralization of phoneme sequence generation. Neural Computation, 10(5), 1277-1297.
Sharma, A., \& Dorman, M. F. (1999). Cortical auditory evoked potential correlates of categorical perception of voice-onset-time. JASA, 106, 1078-1083.

Sidtis, J. J., \& Volpe, B. T. (1988). Selective loss of complex-pitch or speech discrimination after unilateral lesion. Brain and Language, 34, 225-245.

Stevens, N. K., \& Blumstein, S. E. (1981). The search for invariant accoustic correlates of phonetic features. In P. D. Eimas, \& J. L. Miller (Eds.), Perspectives on the study of speech. Hillsdale, NJ: Erlbaum.

Tallal, P., \& Piercy, M. (1974). Developmental aphasia: Rate of auditory processing and selective impairment of consonant perception. Neuropsychologia, 12, 83-93.

Tiitinen, H., May, P., Reinikainen, K., \& Näätänen, R. (1994). Attentive novelty detection is governed by pre-attentive sensory memory. Nature, 372, 90-92.

Tremblay, K., Kraus, N., \& McGee, T. (1998). The time course of auditory perceptuel learning: Neurophysiological changes during speech-sound training. Neuroreport, 9, 33557-33560.

Tzourio-Mazoyer, N., De Schonen, S., Crivello, F., Reutter, B., Aujard, Y., \& Mazoyer, B. (2002). Neural correlates of woman face processing by 2-month-old infants. Neuroimage, 15(2), 454-461.

Vargha-Khadem, F., \& Corballis, M. (1979). Cerebral asymmetry in infants. Brain and Language, 8, 1-9.

Winkler, I., Cowan, N., Csépe, V., Czigler, I., \& Näätänen, R. (1996). Interactions between transient and long-term auditory memory as reflected by the mismatch negativity. Journal of Cognitive Neuroscience, 8(5), 403-415.

Zatorre, R. J., \& Belin, P. (2001). Spectral and temporal processing in human auditory cortex. Cerebral Cortex, 11(10), 946-953.

Zatorre, R. J., Belin, P., \& Penhune, V. B. (2002). Structure and function of auditory cortex: Music and speech. Trends in Cognitve Science, 6(1), 37-46. 